\title{
Leukemia cells are sensitized to temozolomide, carmustine and melphalan by the inhibition of $\mathrm{O}^{6}$-methylguanine-DNA methyltransferase
}

\author{
HAJIME ARAI, TAKAHIRO YAMAUCHI, KANAKO UZUI and TAKANORI UEDA
}

Department of Hematology and Oncology, Faculty of Medical Sciences, University of Fukui, Eiheiji, Fukui 910-1193, Japan

Received August 8, 2014; Accepted April 13, 2015

DOI: $10.3892 / \mathrm{ol} .2015 .3307$

\begin{abstract}
The cytotoxicity of the monofunctional alkylator, temozolomide (TMZ), is known to be mediated by mismatch repair (MMR) triggered by $\mathrm{O}^{6}$-alkylguanine. By contrast, the cytotoxicity of bifunctional alkylators, including carmustine (BCNU) and melphalan (MEL), depends on interstrand crosslinks formed through $\mathrm{O}^{6}$-alkylguanine, which is repaired by nucleotide excision repair and recombination. $\mathrm{O}^{6}$-alkylguanine is removed by $\mathrm{O}^{6}$-methylguanine-DNA methyltransferase (MGMT). The aim of the present study was to evaluate the cytotoxicity of TMZ, BCNU and MEL in two different leukemic cell lines (HL-60 and MOLT-4) in the context of DNA repair. The transcript levels of MGMT, ERCC1, hMLH1 and hMSH2 were determined using reverse transcription-quantitative polymerase chain reaction. In addition, the proliferation was measured using the trypan blue exclusion assay. Drug sensitivity was found to vary between the two cell lines. Treatment of the cells with TMZ, BCNU or MEL in combination with $\mathrm{O}^{6}$-benzylguanine, an MGMT inhibitor, was demonstrated to sensitize the two cell lines to these agents. However, the extent of sensitization was not found to be correlated with the expression levels of MGMT transcripts. Furthermore, the drug sensitivity was also not associated with the transcript levels of ERCC1, hMLH1 and hMSH2. Thus, leukemic cells were sensitized to alkylating agents by the inhibition of MGMT.
\end{abstract}

Correspondence to: Dr Takahiro Yamauchi, Department of Hematology and Oncology, Faculty of Medical Sciences, University of Fukui, 23 Shimoaizuki, Matsuoka, Eiheiji, Fukui 910-1193, Japan E-mail: tyamauch@u-fukui.ac.jp

Abbreviations: $\mathrm{IC}_{50}, 50 \%$ growth-inhibitory concentration; MMR, mismatch repair; MGMT, $\mathrm{O}^{6}$-methylguanine-DNA methyltransferase; NER, nucleotide excision repair; $\mathrm{BG}, \mathrm{O}^{6}$-benzylguanine; TMZ, temozolomide or 3,4-dihydro-3-methyl-4-oxoimidazo (5,1-d)-as-tetrazine-8-carboxamide; BCNU, carmustine or 1,3-bis(2-chloroethyl)-1-nitrosourea; MEL, melphalan

Key words: DNA repair, temozolomide, carmustine, melphalan, $\mathrm{O}^{6}$-benzylguanine

\section{Introduction}

Alkylating agents comprise a major class of chemotherapeutic agents, widely used in various types of cancer, including leukemia $(1,2)$. There are two types of alkylating agents: monofunctional and bifunctional agents. Bifunctional alkylating agents include cyclophosphamide, ifosfamide, melphalan (MEL) and carmustine (BCNU; also known as 1,3-bis(2-chloroethyl)-1-nitrosourea). Monofunctional agents include temozolomide [TMZ; also known as 3,4-dihydro-3-methyl-4-oxoimidazo (5,1-d)-as-tetrazine-8-carboxamide] and dacarbazine (1-3).

Alkylating agents form a variety of DNA adducts in cancer cells, including mono-adducts on $\mathrm{N}^{1}$-alkylguanine, $\mathrm{N}^{3}$-alkyladenine, $\mathrm{N}^{7}$-alkylguanine or $\mathrm{O}^{6}$-alkylguanine, and di-adducts between or within DNA strands (1-4). Bifunctional alkylating agents result in cytotoxicity due to the production of interstrand crosslinks, which are formed through the intermediate production of $\mathrm{O}^{6}$-alkylguanine $(5,6)$. These crosslinks are repaired through nucleotide excision repair (NER) and recombination. By contrast, monofunctional agents generate persistent $\mathrm{O}^{6}$-methylguanine adducts that initiate futile cycling of the DNA mismatch repair (MMR) pathway, which causes DNA double-strand breaks (7-10). Intact MMR is required for the exertion of the cytotoxicity of monofunctional agents. The DNA repair enzyme, $\mathrm{O}^{6}$-methylguanine-DNA methyltransferase (MGMT), repairs $\mathrm{O}^{6}$-alkylguanine adducts $(11,12)$ and reverses the cytotoxicity induced by the two types of alkylating agents.

The cytotoxic effects of alkylating agents are limited by a number of factors, including DNA repair $(2,13,14)$. In the present study, the cytotoxic effects of the bifunctional BCNU and MEL agents, as well as the monofunctional $\mathrm{TMZ}$ agent, were evaluated in relation to DNA repair. The effects were compared in two cultured leukemia cell lines, HL-60 and MOLT-4. In addition, the sensitivity of the cells was manipulated by the addition of an MGMT inhibitor, $\mathrm{O}^{6}$-benzylguanine (BG). The extent of the drug cytotoxicity was analyzed to determine its correlation with DNA repair, including any associations with MGMT, NER and MMR $(15,16)$.

Our previous study demonstrated the important role of MMR in the exertion of the cytotoxicity of monofunctional agent temozolomide (17). Restored MMR sensitized the 
cancer cells to temozolomide. Therefore, the aim of the present study was to evaluate the cytotoxicity of alkylating agents from the viewpoint of MGMT.

\section{Materials and methods}

Chemicals and reagents. BCNU, MEL and BG (all purchased from Sigma-Aldrich, St. Louis, MO, USA) were dissolved in $99 \%$ ethanol immediately prior to use. TMZ (Schering-Plough KK, Osaka, Japan) was dissolved in 100\% dimethyl sulfoxide immediately prior to use.

Cell culture. Human acute myeloid leukemia cell line, HL-60, and human acute T lymphoblastic leukemia cell line, MOLT-4, were used in this study (JCRB Cell Bank, Osaka, Japan). The cells were cultured in RPMI 1640 medium (Life Technologies Japan, Ltd., Tokyo, Japan) in a humidified atmosphere with $5 \% \mathrm{CO}_{2}$ at $37^{\circ} \mathrm{C}$.

Drug treatment and proliferation assay. To evaluate the growth-inhibitory effect of each agent on the two cell lines, the trypan blue exclusion assay was performed $(17,18)$. Briefly, the cells were incubated with various concentrations of TMZ, BCNU or MEL $(10 \mathrm{nM}, 100 \mathrm{nM}, 1 \mu \mathrm{M}$ or $10 \mu \mathrm{M})$, alone or in combination with BG $(10 \mu \mathrm{M})$, for $72 \mathrm{~h}$. Subsequently, the samples were stained with trypan blue (Wako Pure Chemical Industries, Ltd., Osaka, Japan), and the viable cells, which exhibited negative staining, were counted. The $50 \%$ growth-inhibitory concentration $\left(\mathrm{IC}_{50}\right)$ was the concentration at which $50 \%$ of the growth of the untreated cells was inhibited. This value was extrapolated from the growth curve drawn for each drug treatment, with $100 \%$ considered to be the condition of untreated cells.

Reverse transcription-quantitative polymerase chain reaction (RT-qPCR). The transcript levels of MGMT, ERCC1, hMLH1 and hMSH2 were determined by RT-qPCR using the ABI Prism 7700 sequence detection system (Applied Biosystems Life Technologies, Foster City, CA, USA). RT-qPCR was performed according to the method of our previous study (17). ERCC1 is responsible for incision of the damaged DNA strand in the NER pathway, while hMLH1 and hMSH2 are two key factors in the MMR response. For MGMT, the sense primer sequence was 5'-TCCCGTTTTCCAGCAAGAGTC-3', and the antisense sequence was 5'-GGGCTGCTAATTGCTGGTAAGA-3'. The TaqMan probe DNA sequence was FAM-CCAGACA GGTGTTATGGAAGCTGCTGAAG-TAMRA (Mitsubishi Kagaku Bio-Clinical Laboratories, Tokyo, Japan). In addition, the primers for ERCC1, hMLH1 and hMSH2 were purchased from Mitsubishi Kagaku Bio-Clinical Laboratories. The absolute standard curve quantitation method was used for MGMT and ERCC1, and the relative standard curve quantitation method was used for hMLH1 and hMSH2. The values of HL-60 cells were set to 1 and relative values were determined for the MOLT- 4 cells.

Statistical analyses. Graphs were generated using the GraphPad Prism software (version 5.0; GraphPad Software, Inc., San Diego, CA, USA). Spearman's rank correlation
Table I. Drug sensitivities of the two leukemia cell lines.

\begin{tabular}{lrr}
\hline & \multicolumn{2}{c}{$\mathrm{IC}_{50}(\mu \mathrm{M})$} \\
\cline { 2 - 3 } Drugs & HL-60 & MOLT-4 \\
\hline TMZ & 49.0 & 191.5 \\
BCNU & 10.0 & 22.0 \\
MEL & 4.5 & 1.5 \\
TMZ+BG & 4.5 & 169.0 \\
BCNU+BG & 3.0 & 1.4 \\
MEL+BG & 0.3 & 1.4 \\
\hline
\end{tabular}

Cells were incubated with various concentrations of TMZ, BCNU or MEL, with or without $\mathrm{BG}$. The $\mathrm{IC}_{50}$ values were then determined using the trypan blue exclusion assay. TMZ, temozolomide; BCNU, carmustine; MEL, melphalan; $\mathrm{BG}, \mathrm{O}^{6}$-benzylguanine; $\mathrm{IC}_{50}, 50 \%$ growth-inhibitory concentration.

Table II. Sensitization by the addition of BG.

\begin{tabular}{lrr}
\hline & \multicolumn{2}{c}{ Ratio of $\mathrm{IC}_{50}$} \\
\cline { 2 - 3 } Drugs & HL-60 & MOLT-4 \\
\hline TMZ / TMZ+BG & 10.9 & 1.1 \\
BCNU / BCNU+BG & 3.3 & 15.7 \\
MEL / MEL+BG & 15.0 & 1.1 \\
\hline
\end{tabular}

Ratios of $\mathrm{IC}_{50}$ values $=\left(\mathrm{IC}_{50}\right.$ value of TMZ, BCNU or MEL treatment) / $\left(\mathrm{IC}_{50}\right.$ value of TMZ, BCNU or MEL treatment in combination with BG). TMZ, temozolomide; BCNU, carmustine; MEL, melphalan; $\mathrm{BG}, \mathrm{O}^{6}$-benzylguanine; $\mathrm{IC}_{50}, 50 \%$ growth-inhibitory concentration.

was used for determination of any correlation between two parameters. All statistical analyses were performed using Microsoft Excel 2007 (Microsoft Corporation, Redmond, WA, USA). $\mathrm{P}<0.05$ was considered to indicate a statistically significant difference.

\section{Results}

Growth-inhibitory effects of the alkylating agents. The growth-inhibitory effects of TMZ, BCNU and MEL were evaluated in the HL-60 and MOLT- 4 cells. Overall, the sensitivity of the two cell lines to these agents varied; however, treatment with MEL appeared to be the most effective in inhibiting the cell growth (Table I).

Inhibition of MGMT by BG. The cytotoxic effect of alkylating agents is generally reduced by DNA repair in cancer cells (3-6). Upon treatment of the HL-60 and MOLT-4 cells with TMZ, BCNU or MEL in the presence of BG, an MGMT inhibitor, the two cell lines were apparently sensitized to all these agents (Table I). However, the extent of sensitization varied among the drugs in the two cell lines (Table II; Fig. 1). $\mathrm{BG}$ was not found to be cytotoxic to cells in the previous study (17). 

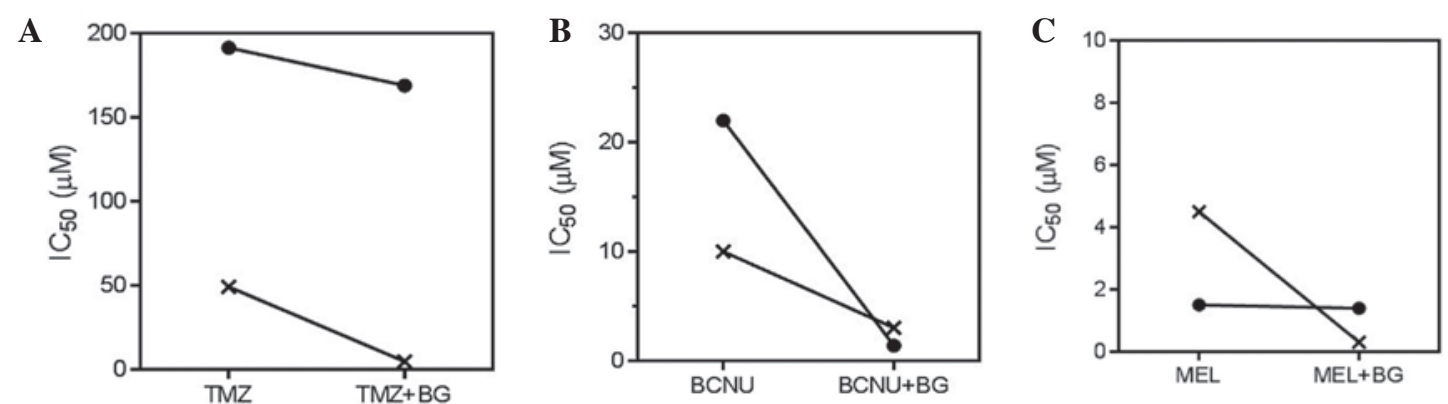

Figure 1. Changes in the $\mathrm{IC}_{50}$ values following the addition of BG. The cellular sensitivity to (A) TMZ, (B) BCNU or (C) MEL were determined in the HL-60 and MOLT- 4 cells in the presence or absence of BG using the trypan blue exclusion assay. Values are expressed as the mean of at least three independent experiments. TMZ, temozolomide; $\mathrm{BCNU}$, carmustine; MEL, melphalan; $\mathrm{BG}, \mathrm{O}^{6}$-benzylguanine; $\mathrm{IC}_{50}, 50 \%$ growth-inhibitory concentration.
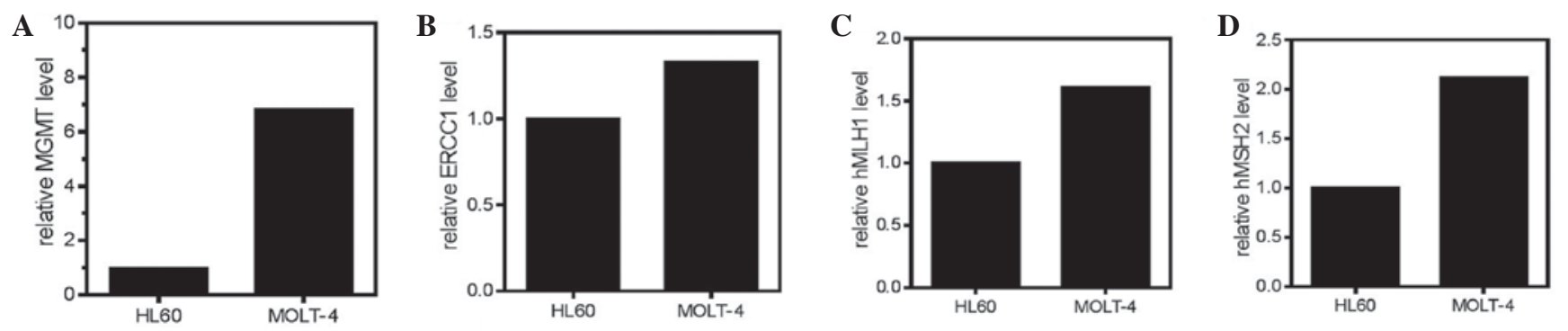

Figure 2. DNA repair-associated transcripts. Relative transcript levels of (A) MGMT, (B) ERCC1, (C) hMLH1 and (D) hMSH2 in the HL-60 and MOLT-4 cells, measured by using reverse transcription-quantitative polymerase chain reaction. The values of HL-60 cells were set to 1. MGMT, $\mathrm{O}^{6}$-methylguanine-DNA methyltransferase.

A

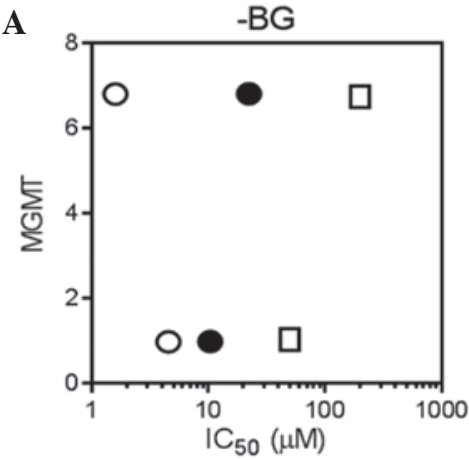

B

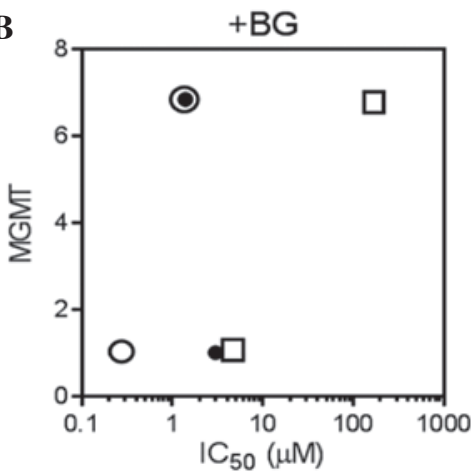

C

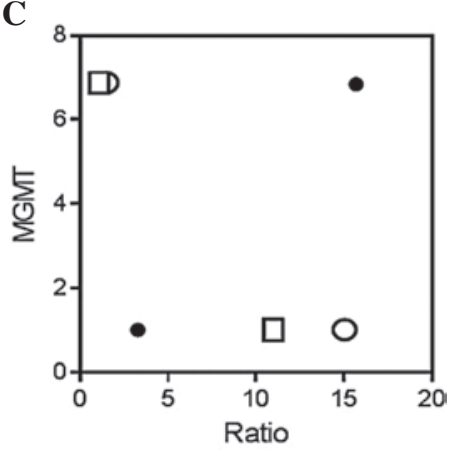

Figure 3. Correlation between MGMT transcript levels and drug sensitivity (in terms of IC 50 values) in HL-60 and MOLT-4 cells. Cells were incubated for $72 \mathrm{~h}$ with various concentrations of TMZ, BCNU or MEL, (A) with or (B) without a minimally toxic concentration of BG (10 $\mu \mathrm{M}$ ). The growth inhibition effects were determined using the trypan blue exclusion assay. Values are expressed as the mean of at least three independent experiments. (C) The ratio between the $\mathrm{IC}_{50}$ values of TMZ/BCNU/MEL to the values of TMZ/BCNU/MEL with BG was plotted against the MGMT transcript levels in each cell line. (A): P>0.99; (B): P>0.99 (Spearman's rank correlation). circle, MEL; filled circle, BCNU; square, TMZ. MGMT, O6 - methylguanine-DNA methyltransferase; TMZ, temozolomide; BCNU, carmustine; MEL, melphalan; $\mathrm{BG}, \mathrm{O}^{6}$-benzylguanine; $\mathrm{IC}_{50}, 50 \%$ growth-inhibitory concentration.

Transcript levels of DNA repair-associated genes. The transcript levels of MGMT, ERCC1, hMLH1 and hMSH2 were determined in the two cell lines (Fig. 2), and were found to be different between the two cell lines. Associations between these expression levels and the drug sensitivity were also investigated (Figs. 3 and 4). Alkylguanine is repaired primarily by MGMT $(12,14,19)$. However, no apparent correlation was observed between the expression levels of MGMT and the drug sensitivity in the presence or absence of $\mathrm{BG}$ (Fig. 3A and B). Furthermore, the ratio of the $\mathrm{IC}_{50}$ value of each agent over the $\mathrm{IC}_{50}$ value of each agent $+\mathrm{BG}$ was calculated (Table II). These values were plotted against the MGMT transcript levels (Fig. 3C). It was expected that the ratio may be higher in the cell line with a higher MGMT transcript level. However, the ratio was not correlated with the MGMT expression levels (Fig. 3C). This indicated that sensitization through the inhibition of MGMT could not be predicted based on the levels of MGMT transcript in these cell lines.

BCNU-mediated interstrand crosslinks are repaired by NER and recombination, while $\mathrm{O}^{6}$-alkylguanine is repaired by NER (20). The ERCC1-XPF heterodimer is an endonuclease that is involved in the NER pathway (15). In the present study, the expression levels of ERCC1 were not found to be correlated to the drug sensitivity (Fig. 4A). No correlation was observed 
A

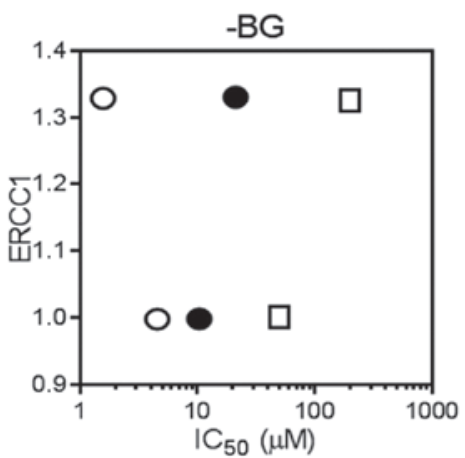

D

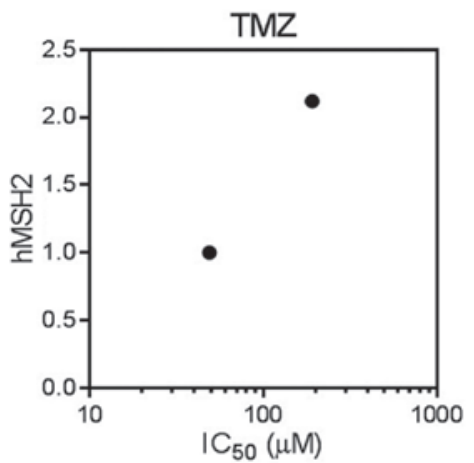

B

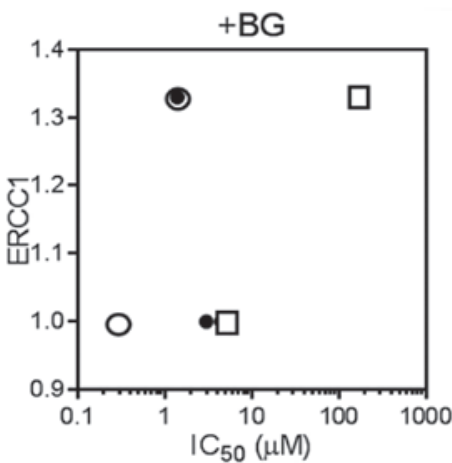

E

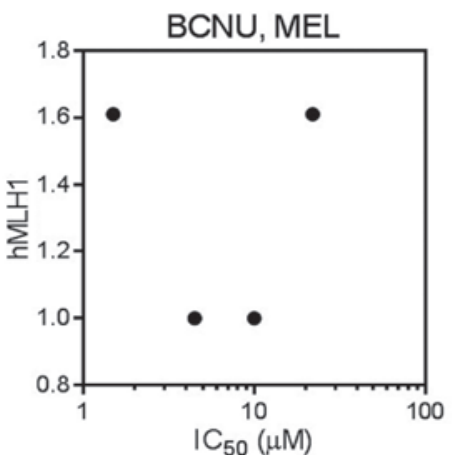

C

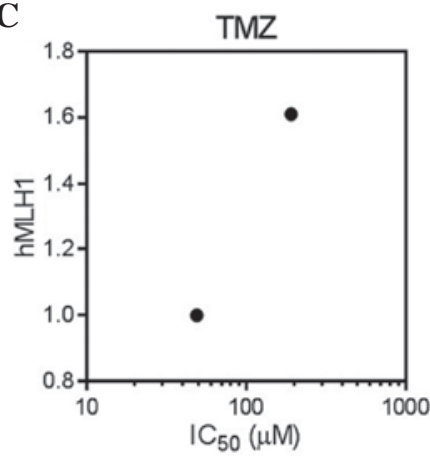

F

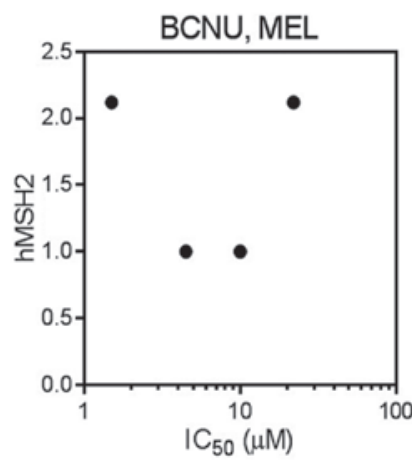

Figure 4. Correlation between ERCC1/hMLH1/hMSH2 transcript levels and $\mathrm{IC}_{50}$ values of BCNU/TMZ/MEL in HL-60 and MOLT-4 cells. The IC ${ }_{50}$ values of TMZ, BCNU and MEL, (A) with and (B) without a minimally toxic concentration of BG (10 $\mu \mathrm{M})$ were plotted against ERCC1 transcript levels in the HL-60 and MOLT-4 cells (circle, MEL; filled circle, BCNU; square, TMZ). The $\mathrm{IC}_{50}$ values of TMZ were plotted against the (C) hMLH1 and (D) hMSH2 transcript levels of the HL-60 and MOLT-4 cells. The $\mathrm{IC}_{50}$ values of BCNU and MEL were plotted against the (E) hMLH1 and (F) hMSH2 transcript levels of the HL-60 and MOLT-4 cells. TMZ, temozolomide; BCNU, carmustine; MEL, melphalan; BG, $\mathrm{O}^{6}$-benzylguanine; $\mathrm{IC}_{50}, 50 \%$ growth-inhibitory concentration.

using the $\mathrm{IC}_{50}$ values for the co-treatment with $\mathrm{BG}$, which is an inhibitor of the activity of MGMT (Fig. 4B). Deficiency in MMR is known to result in cellular insensitivity to TMZ (10), while MMR is suggested to be involved in crosslink repair induced by BCNU (21). Based on these previous observations, a lower MMR function may be associated with lower sensitivity to a monofunctional TMZ, whereas a higher MMR function may confer the higher resistance to bifunctional BCNU and MEL. However, in the present study, no association was detected between the expression levels of the MMR-associated hMLH1/hMSH2 and the sensitivity to these two agents (Fig. 4C-F).

\section{Discussion}

The MMR response triggered by $\mathrm{O}^{6}$-alkylguanine-mediated mismatch is indispensable to the exertion of the cytotoxicity of TMZ. Interstrand crosslinks are formed through the intermediate production of $\mathrm{O}^{6}$-alkylguanine in the process of the cytotoxic action of BCNU and MEL (22). Therefore, MGMT is closely associated with the mechanisms of resistance to these agents. In the present study, two different leukemia cell lines were sensitized to monofunctional (TMZ) and bifunctional alkylating agents (BCNU and MEL) by an MGMT inhibitor, $\mathrm{BG}$. The results indicated that $\mathrm{O}^{6}$-alkylguanine was the major cytotoxic lesion generated by these alkylators. However, the sensitivity of the cells to these agents and the extent of the sensitization by BG were not found to be correlated with the MGMT transcript levels.

A previous study evaluated the role of $\mathrm{BG}$ in restoring TMZ sensitivity in patients with recurrent or progressive
TMZ-resistant malignant glioma in a phase II trial (23). Both TMZ and BG were administered on day 1 of a 28-day treatment cycle. Patients were administered a 1-h BG infusion at a dose of $120 \mathrm{mg} / \mathrm{m}^{2}$, immediately followed by a 48-h infusion at a dose of $30 \mathrm{mg} / \mathrm{m}^{2}$. TMZ was administered orally within $60 \mathrm{~min}$ after the end of the 1-h BG infusion at a dose of $472 \mathrm{mg} / \mathrm{m}^{2}$. Out of the 66 patients treated with TMZ and BG, only six patients responded to the treatment, indicating that the efficacy of this combination was limited. In addition, the Children Oncology Group evaluated the combination treatment with BCNU and BG in pediatric patients with central nervous system tumors in a phase I study (24). The toxicity of this treatment was evaluated in 25 patients, and the maximum tolerated dose of BCNU administered with BG $\left(120 \mathrm{mg} / \mathrm{m}^{2}\right)$ was $58 \mathrm{mg} / \mathrm{m}^{2}$. Furthermore, the response to this treatment was evaluated in 24 patients, and only six patients were found to present stable disease, while one patient exhibited a minor response (24). A study by Hegi et al investigated the association between MGMT silencing and the survival of patients with glioblastoma, treated with radiotherapy alone or radiotherapy combined with TMZ (25). The MGMT promoter was methylated in $45 \%$ of the 206 assessed cases and the methylation was an independent favorable prognostic factor (25). The authors concluded that patients with glioblastoma containing a methylated MGMT promoter benefited from TMZ, whereas patients without a methylated MGMT promoter did not benefit from the treatment (25). Therefore, the critical role of MGMT was demonstrated in the therapeutic outcome of alkylator-based cancer treatment $(25,26)$. However, the efficacy of combination treatment with BG has not yet been confirmed clinically. 
A number of proteins, including hMLH1 and hMSH2, participate in the process of MMR, which involves the mismatch recognition, excision of the DNA-containing error and resynthesis of the correct DNA $(3,10)$. Intact MMR is required for the exertion of the cytotoxicity of TMZ, while interstrand crosslinks formed by bifunctional agents are, in part, repaired by MMR $(21,27)$. Our previous study evaluated TMZ cytotoxicity in a BCNU-resistant variant leukemia cell line, in comparison with a BCNU-sensitive cell line (17). The study identified that the BCNU-resistant cells were more sensitive to TMZ compared with the BCNU-sensitive cells (17). In addition, the BCNU-resistant cells possessed increased hMLH1 and hMSH2 transcript levels (17). However, when the cells were transfected with shRNA against hMLH1, the sensitivity to TMZ was partially reversed. Therefore, the study suggested inverse roles of MMR on the cytotoxicity between TMZ and BCNU (17). The present study investigated only two leukemia cell lines and, therefore, it may be difficult to clarify the role of MMR in the sensitivity of leukemia cells to alkylating agents.

In conclusion, the present study evaluated the cytotoxic effects of monofunctional and bifunctional alkylating agents in relation to DNA repair in two different leukemic cell lines. The results revealed that the inhibition of MGMT appeared to sensitize the two leukemia cell lines to TMZ, BCNU and MEL. However, no correlation was identified between the drug sensitivity and MGMT transcript levels.

\section{References}

1. Tew KD, Colvin M and Chabner BA: Alkylating agents. In: Cancer Chemotherapy and Biotherapy: Principles and Practice. Chabner BA and Longo DL (eds). Lippincott-Raven Publishers, Philadelphia, pp297-317, 1996.

2. Fu D, Calvo JA and Samson LD: Balancing repair and tolerance of DNA damage caused by alkylating agents. Nat Rev Cancer 12: 104-120, 2012.

3. Geleziunas R, McQuillan A, Malapetsa A, Hutchinson M, Kopriva D, Wainberg MA, Hiscott J, Bramson J and Panasci L: Increased DNA synthesis and repair-enzyme expression in lymphocytes from patients with chronic lymphocytic leukemia resistant to nitrogen mustards. J Natl Cancer Inst 83: 557-564, 1991.

4. Drabløs F, Feyzi E, Aas PA, Vaagbø CB, Kavli B, Bratlie MS, Peña-Diaz J, Otterlei M, Slupphaug G and Krokan HE: Alkylation damage in DNA and RNA - repair mechanisms and medical significance. DNA Repair (Amst) 3: 1389-1407, 2004.

5. Wiencke JK and Wiemels J: Genotoxicity of 1,3-bis(2-chloroethyl) -1-nitrosourea (BCNU). Mutat Res 339: 91-119, 1995.

6. Povirk LF and Shuker DE: DNA damage and mutagenesis induced by nitrogen mustards. Mutat Res 318: 205-226, 1994.

7. Friedman HS, Kerby $\mathrm{T}$ and Calvert H: Temozolomide and treatment of malignant glioma. Clin Cancer Res 6: 2585-2597, 2000.

8. Stupp R, Gander M, Leyvraz S and Newlands E: Current and future developments in the use of temozolomide for the treatment of brain tumours. Lancet Oncol 2: 552-560, 2001.

9. Mason WP and Cairncross JG: Drug Insight: Temozolomide as a treatment for malignant glioma - impact of a recent trial. Nat Clin Pract Neurol 1: 88-95, 2005.

10. Fink D, Aebi S and Howell SB: The role of DNA mismatch repair in drug resistance. Clin Cancer Res 4: 1-6, 1998.
11. Fan $\mathrm{CH}$, Liu WL, Cao $\mathrm{H}$, Wen $\mathrm{C}$, Chen L and Jiang G: $\mathrm{O}^{6}$-methylguanine DNA methyltransferase as a promising target for the treatment of temozolomide-resistant gliomas. Cell Death Dis 4: e876, 2013

12. Fahrer J and Kaina B: $\mathrm{O}^{6}$-methylguanine-DNA methyltransferase in the defense against $\mathrm{N}$-nitroso compounds and colorectal cancer. Carcinogenesis 34: 2435-2442, 2013.

13. Chaney SG and Sancar A: DNA repair: Enzymatic mechanisms and relevance to drug response. J Natl Cancer Inst 88: 1346-1360, 1996.

14. Panasci L, Paiement JP, Christodoulopoulos G, Belenkov A, Malapetsa A and Aloyz R: Chlorambucil drug resistance in chronic lymphocytic leukemia: The emerging role of DNA repair. Clin Cancer Res 7: 454-461, 2001.

15. Bowden NA: Nucleotide excision repair: Why is it not used to predict response to platinum-based chemotherapy? Cancer Lett 346: 163-171, 2014.

16. Martin SA, Lord CJ and Ashworth A: Therapeutic targeting of the DNA mismatch repair pathway. Clin Cancer Res 16: 5107-5113, 2010.

17. Yamauchi T, Ogawa M and Ueda T: Carmustine-resistant cancer cells are sensitized to temozolomide as a result of enhanced mismatch repair during the development of carmustine resistance. Mol Pharmacol 74: 82-91, 2008.

18. Yamamoto S, Yamauchi T, Kawai Y, Takemura $H$, Kishi S, Yoshida A, Urasaki Y, Iwasaki $\mathrm{H}$ and Ueda T: Fludarabine-mediated circumvention of cytarabine resistance is associated with fludarabine triphosphate accumulation in cytarabine-resistant leukemic cells. Int J Hematol 85: 108-115, 2007.

19. Esteller M, Garcia-Foncillas J, Andion E, Goodman SN Hidalgo OF, Vanaclocha V, Baylin SB and Herman JG: Inactivation of the DNA-repair gene MGMT and the clinical response of gliomas to alkylating agents. N Engl J Med 343; $1350-1354,2000$.

20. Shrivastav N, Li D and Essigmann JM: Chemical biology of mutagenesis and DNA repair: Cellular responses to DNA alkylation. Carcinogenesis 31: 59-70, 2010.

21. Fiumicino S, Martinelli S, Colussi C, Aquilina G, Leonetti C, Crescenzi M and Bignami M: Sensitivity to DNA cross-linking chemotherapeutic agents in mismatch repair-defective cells in vitro and in xenografts. Int J Cancer 85: 590-596, 2000.

22. Loeber R, Michaelson E, Fang Q, Campbell C, Pegg AE and Tretyakova N: Cross-linking of the DNA repair protein $\mathrm{O}^{6}$-alkylguanine DNA alkyltransferase to DNA in the presence of antitumor nitrogen mustards. Chem Res Toxicol 21: 787-795, 2008.

23. Quinn JA, Jiang SX, Reardon DA, Desjardins A, Vredenburgh JJ, Rich JN, Gururangan S, Friedman AH, Bigner DD, Sampson JH, et al: Phase II trial of temozolomide plus $\mathrm{O}^{6}$-benzylguanine in adults with recurrent, temozolomide-resistant malignant glioma. J Clin Oncol 27: 1262-1267, 2009.

24. Adams DM, Zhou T, Berg SL, Bernstein M, Neville K and Blaney SM; Children's Oncology Group: Phase 1 trial of $\mathrm{O}^{6}$-benzylguanine and $\mathrm{BCNU}$ in children with CNS tumors: A Children's Oncology Group study. Pediatr Blood Cancer 50: 549-553, 2008

25. Hegi ME, Diserens AC, Gorlia T, Hamou MF, de Tribolet N, Weller M, Kros JM, Hainfellner JA, Mason W, Mariani L, et al: MGMT gene silencing and benefit from temozolomide in glioblastoma. N Engl J Med 352: 997-1003, 2005.

26. Balaña C, Ramirez JL, Taron M, Roussos Y, Ariza A, Ballester R, Sarries C, Mendez P, Sanchez JJ and Rosell R: $\mathrm{O}^{6}$-methyl-guanine-DNA methyltransferase methylation in serum and tumorDNA predicts response to 1,3-bis(2-chloroethyl)-1-nitrosourea but not to temozolamide plus cisplatin in glioblastoma multiforme. Clin Cancer Res 9: 1461-1468, 2003.

27. Aquilina G, Ceccotti S, Martinelli S, Hampson R and Bignami M: $\mathrm{N}$-(2-chloroethyl)-N'-cyclohexyl-N-nitrosourea sensitivity in mismatch repair-defective human cells. Cancer Res 58: 135-141, 1998. 